\title{
Variable Temperature Performance of a Si(Li) Detector Stack
}

\author{
G. Scott Hubbard, Robert E. McMurray, Jr., R.G. Keller, and P.F. Wercinski \\ NASA-Ames Research Center \\ Moffett Field, CA 94035-1000 \\ J.T. Walton, Y.K. Wong \\ Lawrence Berkeley Laboratory \\ Berkeley, CA 94720 \\ NASA-TM-111912
}

\begin{abstract}
New experimental data is presented which displays ${ }^{137} \mathrm{Cs}$ resolution of both single $\mathrm{Si}(\mathrm{Li})$ devices and a detector stack $2 \mathrm{~cm}$ in height as a function of temperature ( $85 \mathrm{~K} \leq \mathrm{T} \leq 245 \mathrm{~K})$. We also discuss variations in photopeak shape which indicate that detector charge collection may be temperature dependent over the range of interest.
\end{abstract}

\section{INTRODUCTION}

We have previously reported laboratory experimental data and Monte Carlo analysis for a $\mathrm{Si}(\mathrm{Li})$ gamma-ray detector stack $[1,2]$. This configuration is intended to provide moderate resolution [full-width at half-maximum (FWHM) 5-10 keV @ $1 \mathrm{MeV})$ ] over a broad temperature range ( $<90 \mathrm{~K}$ to $230 \mathrm{~K}$ ). This temperature range is consistent with the needs of spaceborne applications using ambient (passive) cooling for orbiting platforms $(\geq 100 \mathrm{~K}$ ) or for Mars surface operations $(130 \mathrm{~K}-230 \mathrm{~K})$. Such an approach provides an attractive alternative to the poorer resolution of scintillators $(\sim 60 \mathrm{keV}$ $@ 1.3 \mathrm{MeV}$ ) and the cryogenic cooling requirements of HPGe $(\leq 100 \mathrm{~K})$.

For simplicity in the earlier work, experimental resolution data was collected at $175 \mathrm{~K}$ [1]. DC leakage current indicated that acceptable device performance (FWHM $<10 \mathrm{keV}$ @ $662 \mathrm{keV}$ ) could be expected up to $\sim 230 \mathrm{~K}$, where leakage current was $\sim 10 \mathrm{nA}$. However, it is well known that leakage current is only one contributor to detector performance, and it has been suggested that detector performance often degrades as a function of temperature faster than indicated by DC measurements. In addition, the literature provides little experimental data on the performance of $\mathrm{Si}(\mathrm{Li})$ detectors at temperatures above that of liquid nitrogen $(\sim 77 \mathrm{~K})$ [1]. This current work addresses that absence of data, providing variable temperature resolution measurements from $85 \mathrm{~K}$ to $245 \mathrm{~K}$.

\section{EXPERIMENTAL PROCEDURE}

\section{A. Device Testing}

A total of $8 \mathrm{Si}(\mathrm{Li})$ devices were tested in a variable temperature cryostat constructed for this work. We evaluated 6 devices $5 \mathrm{~mm}$ thick and 2 devices $1 \mathrm{~cm}$ thick; all detectors have a $3 \mathrm{~cm}^{2}$ active area. These devices were fabricated using standard lithium drifting techniques employed at the Lawrence Berkeley Laboratory (LBL) silicon detector laboratory, with no special attention to elevated temperature operation.

All devices feature a standard grooved structure, however the $1 \mathrm{~cm}$ thick detectors had their exterior annulus reduced in height to accommodate electrical connections to the center active area more easily. We followed the convention of applying positive bias to the $\mathrm{Li}^{+}$contact, and obtaining the detector signal from the Au Schottky barrier side. In our previous work, we typically fabricated detector stacks of four $5 \mathrm{~mm} \mathrm{Si}(\mathrm{Li})$ devices to create an active absorption length of $2 \mathrm{~cm}$. In this current work, we were able to obtain several $1 \mathrm{~cm}$ thick devices, which simplified the task of assembling a $2 \mathrm{~cm}$ stack. Future plans call for tests of a stack of four $1 \mathrm{~cm}$ devices.

Testing conditions were standardized except as noted elsewhere. Both individual detectors and detector stacks were biased to 600 volts and data collection times were 500 seconds. ${ }^{137} \mathrm{Cs}(662 \mathrm{keV})$ was used throughout as the $\gamma$-ray source. As noted in the previous work, this isotope was selected because Compton scattering is the primary mechanism of energy deposition for $\mathrm{Si}$ in this energy range and therefore represents a rigorous test of the full energy photopeak detection. As discussed later, an additional parameter varied was amplifier shaping time.

\section{B. Variable Temperature Cryostat Configuration}

Figure 1 displays the elements of the cryostat configuration. A key experimental challenge was to develop a wide-range, variable temperature cryostat which is both low noise and allows for rapid thermal cycling. Low noise temperature control $(90 \mathrm{~K} \leq \mathrm{T} \leq 245 \mathrm{~K})$ was achieved via power input and control measurement at the cold finger. This approach eliminated undesirable noise introduced when the controller heater was placed near the detector cold plate inside the vacuum shroud. Only temperature is measured at the detector, using a calibrated Si diode. In order to reach higher temperatures and also provide stable temperature control, we employed a braid contact to the $\mathrm{LN}_{2}$ to provide a thermal impedance to the cold sink. Rapid thermal cycling is achieved 
by submerging the cold finger in $\mathrm{LN}_{2}$ until low temperature is reached at the detector cold plate $(85 \mathrm{~K})$, then withdrawing the apparatus until only the braid connects the cryostat with the $\mathrm{LN}_{2}$.

The inset of Fig. 1 displays the internal detector mounting scheme. We show spring loaded detector fasteners which were constructed to provide good thermal contact through constant pressure and also prevent breakage as metallic parts shrink at low temperatures. Also visible is the aluminum ${ }^{137} \mathrm{Cs}$ source holder which guaranteed a constant detector/source geometry and also blocked the unwanted beta particle continuum.

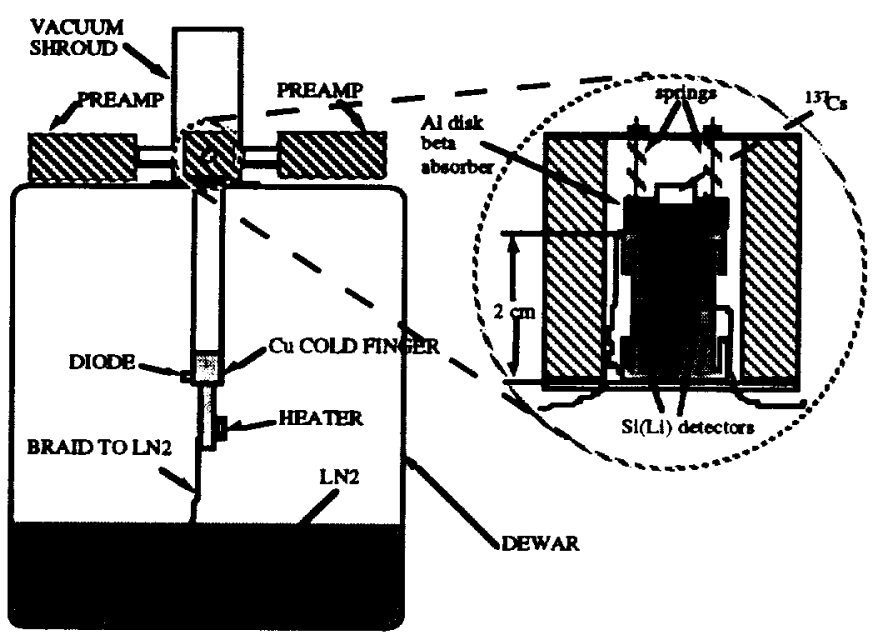

Figure 1. Experimental Apparatus. Insert shows detector configuration in vacuum shroud.

\section{Electronics}

All measurements in this current work were conducted using either the output from a single device or the summed mode for detector stacks. As shown in Figure 2, the signal output from the stack elements was combined from all devices before the vacuum feedthrough to the preamplifier. The spectroscopy amplifier, high voltage power supply and multichannel analyzer (MCA) used are standard commercially available units.

Selection and optimization of the preamplifier (provided by LBL) was driven by the need to compensate for competing noise sources across the broad temperature range of interest. For example, the best electronic noise performance is typically obtained by using a DC-coupled preamp, often with an optofeedback circuit and cooled FET. In our preliminary testing we found that in the range of $150 \mathrm{~K} \leq \mathrm{T} \leq 200 \mathrm{~K}$ leakage currents became unacceptable for DC-coupled preamps. Alternatively, AC-coupled preamps, which are far more tolerant of high leakage currents, are customarily designed for high capacitance surface barrier devices used for charged particle spectroscopy and therefore not optimized for a $\mathrm{Si}(\mathrm{Li})$ stack capacitance of $\sim 10 \mathrm{pf}$. Our choice for the current experiments was an AC-coupled preamp with input circuit and FET selected for the lower capacitance and leakage current varying from a few pA to $>50 \mathrm{nA}$. In future work we believe more improvement in the electronic noise component is possible.

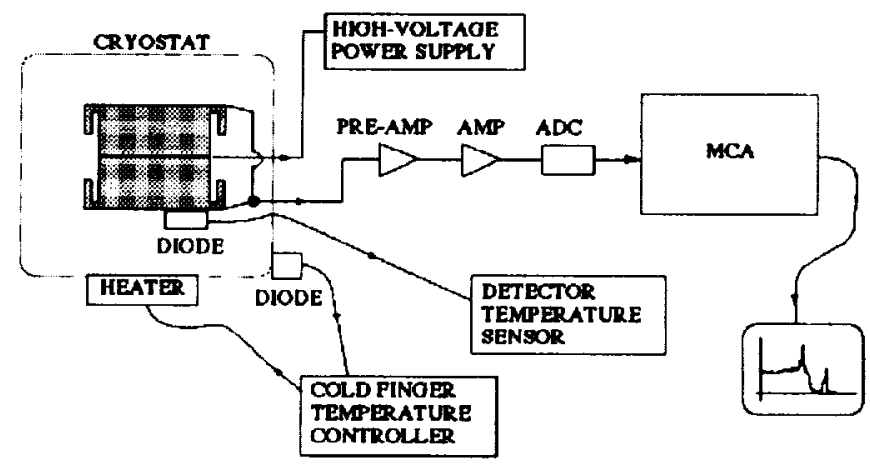

Figure 2. Signal Processing and Temperature Controllers. Control heater and diode are mounted on cold finger as shown in Figure 1.

\section{RESULTS AND DISCUSSION}

\section{A. Initial Device Testing and Selection}

To establish suitability of $\mathrm{Si}(\mathrm{Li})$ devices over the range of interest, and to provide a basis of comparison with our previous leakage current measurements, five $\mathrm{Si}(\mathrm{Li})$ devices were selected on the basis of room temperature noise performance from a larger group of detectors. (A sixth detector was discarded on the basis of excess leakage at ambient.)

Results of ${ }^{137} \mathrm{Cs}$ resolution measurements vs. temperature for all these detectors are shown in Figure 3. Electronic and environmental noise performance was not optimized, rather the results were used for qualitative screening only. As can be seen, the resolution variation is relatively small $( \pm 20 \%)$ among devices up to temperatures of approximately $200 \mathrm{~K}$. In addition, such detectors individually met the key resolution criteria of $<10 \mathrm{keV}$ up to $230 \mathrm{~K}$. Based on these results we concluded that even without special fabrication or selection procedures, devices on hand at LBL should yield stacks which respond consistently to variable temperature operation.

\section{B. Detector Stack Results}

As noted earlier, several $1 \mathrm{~cm}$ thick devices were available, which simplified the construction of a $2 \mathrm{~cm}$ stack. This absorption length was chosen initially as appropriate to test rigorously the stack concept using a $662 \mathrm{keV}$ source $\left({ }^{137} \mathrm{Cs}\right)$. Figure 4 shows the resolution (FHWM) of ${ }^{137} \mathrm{Cs}$ photopeak vs. $\mathrm{T}$ for the $2 \mathrm{~cm}$ stack. (Amplifier shaping time was optimized throughout data collection - see Sec. III C). 


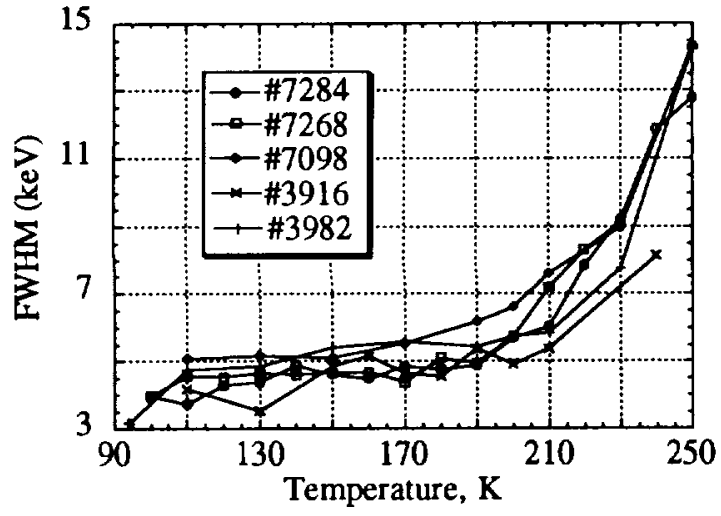

Figure 3. Resolution vs. temperature at $662 \mathrm{keV}$, five test detectors.

Data analysis was conducted with standard MCA output and an off-line gaussian fitting routine. It was found that analysis of the data using a gaussian peak fit yielded somewhat more consistent results than the commercially provided FWHM measurement algorithm resident in the MCA. The results displayed in Figure 4 were fitted with a constant background superimposed with an exponential curve. A detector stack resolution of $3.92 \mathrm{keV}$ up to $150 \mathrm{~K}$ was obtained through this analysis. Electronic noise as determined by precision pulser is $3.68 \mathrm{keV}$ for the same temperature range. If one assumes that noise contributions sum in quadrature, one can determine that the contribution of the $\mathrm{Si}(\mathrm{Li})$ device is

$$
\sqrt{(3.92 \mathrm{keV})^{2}-(3.68 \mathrm{keV})^{2}}=1.4 \mathrm{keV} \text {. }
$$

This value is approximately the same as the noise of charge production statistics in Si at an energy of $662 \mathrm{keV}$ and a Fano factor of 0.15 . At higher temperatures this relationship does not hold. These results suggest that "normal" $\mathrm{Si}(\mathrm{Li})$ device performance may be obtained up to $\sim 150 \mathrm{~K}$, but that an excess noise mechanism begins to dominate at higher temperatures. A measurement of the DC leakage current of this stack from $100 \mathrm{~K}$ to $240 \mathrm{~K}$ demonstrates that the current remains below $1 \mathrm{nA}$ up to $220 \mathrm{~K}$. The noise contribution from this current is small compared to the measured FWHM. Further evaluation of both $\mathrm{DC}$ and $\mathrm{AC}$ components of this noise contribution is underway.

\section{Optimization of Shaping time.}

During testing and evaluation of both individual devices and detector stacks, asymmetric $662 \mathrm{keV}$ photopeaks were observed at elevated temperatures. Figure 5 a-d shows a typical variation in peak shape vs. increasing $\mathrm{T}$ for a stack of four, $5 \mathrm{~mm}$ detectors for fixed amplifier shaping times $\left(\tau_{\mathrm{s}}\right)$.
The asymmetry was consistently observed to be characterized by counts which accumulated as the well-known "tail" on the lower energy side of the peak.

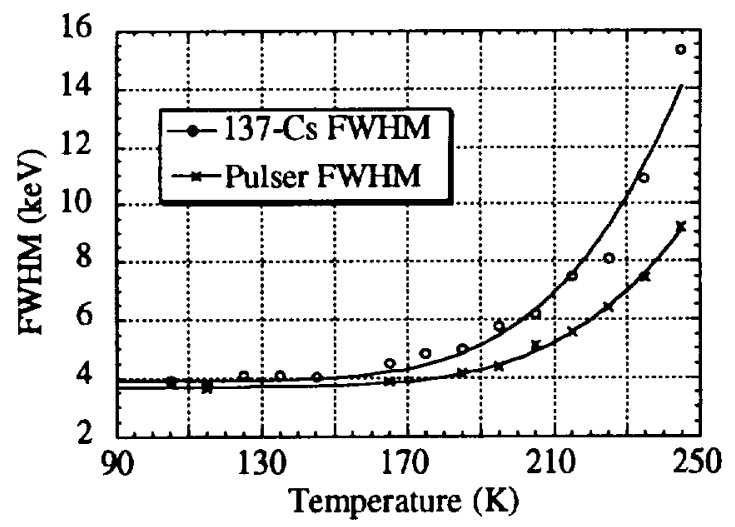

Figure 4. FWHM resolution of ${ }^{137} \mathrm{Cs}$ photopeak using a $\mathrm{Si}(\mathrm{Li})$ stack $2 \mathrm{~cm}$ in height. $\tau_{\mathrm{s}}$ was adjusted for optimum peak shape at each temperature

As shown in Figure 6 a-c, a symmetrical gaussian peak shape could be restored by using progressively longer $\tau_{\mathbf{s}}$. The results in Figure 6 were obtained from a single detector, but are consistent with all stack measurements. Such device behavior is generally associated with charge trapping centers which produce so-called "slow" pulses. These slow pulses can then be collected into the full-energy photopeaks by lengthening the amplifier shaping time.

For the $\mathrm{Si}(\mathrm{Li})$ stack results shown in Fig. 4, optimum resolution was obtained by adjusting the amplifier shaping time as a function of temperature. Figure 7 shows the resolution of the $2 \mathrm{~cm}$ stack as function of amplifier shaping time for $\sim 85 \mathrm{~K}$ and $\sim 200 \mathrm{~K}$. In this figure, the fitted curves shown are only an aid to the reader; no functional relationship is implied. As can be seen, the optimum $\tau_{\mathbf{s}}$ for minimum FWHM shifts by a factor of $>2$. During most measurements over the entire temperature range, only three different $\tau_{\mathrm{s}}$ values were required: $1.5 \mu \mathrm{S}, 3 \mu \mathrm{S}$ and $5 \mu \mathrm{S}$. For a given $\tau_{\mathrm{S}}$, very little peak shift was observed. 


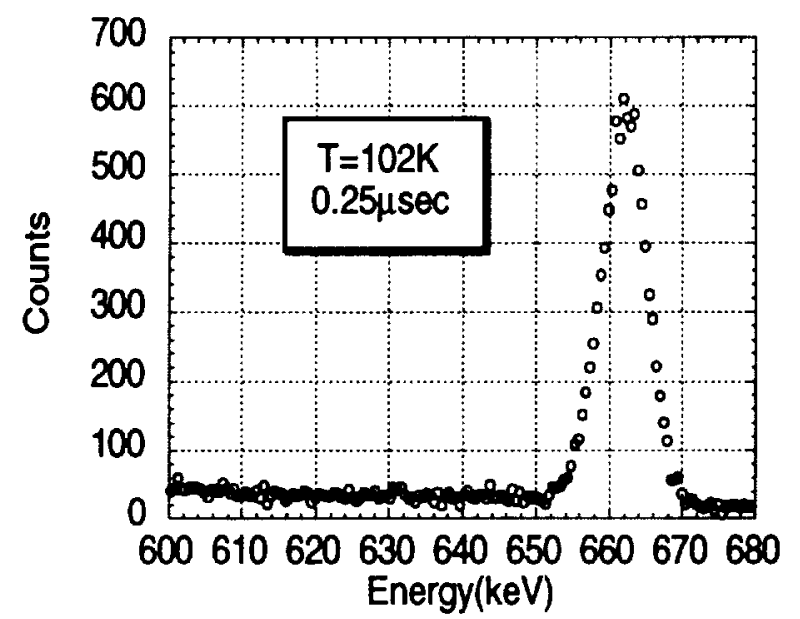

Figure 5(a)

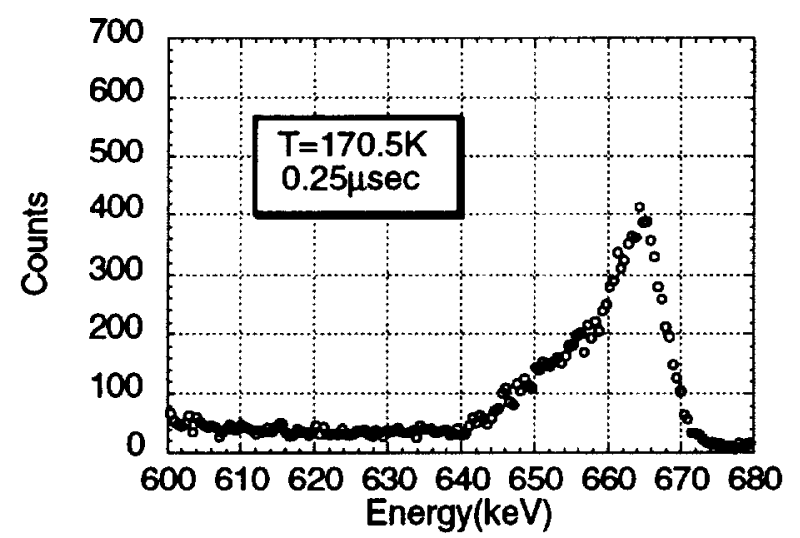

Figure 5(b)

Figure 5. Degradation in photopeak shape as a function of temperature for a single shaping time $(0.25 \mu \mathrm{S})$. Stack of four $5 \mathrm{~mm}$ thick devices selected from those shown in Figure 3.

\section{SUMMARY AND CONCLUSIONS}

In summary the $S i(L i)$ stack measured exhibits the required resolution over the temperature range of interest. We have observed a FWHM for $662 \mathrm{keV}$ of $\leq 5 \mathrm{keV}$ for $\mathrm{T} \leq 200 \mathrm{~K}$ and $\leq 10 \mathrm{keV}$ for $\mathrm{T} \leq 230 \mathrm{~K}$. To obtain an optimized peak shape requires longer $\tau_{\mathrm{s}}$ as device temperature increases. As described earlier, the most likely explanation for this phenomenon is that regions of non-uniform charge collection appear at elevated temperatures and result in charge trapping. Extending $\tau_{s}$ allows "slow" trapped charge to be collected in the full energy photopeak.

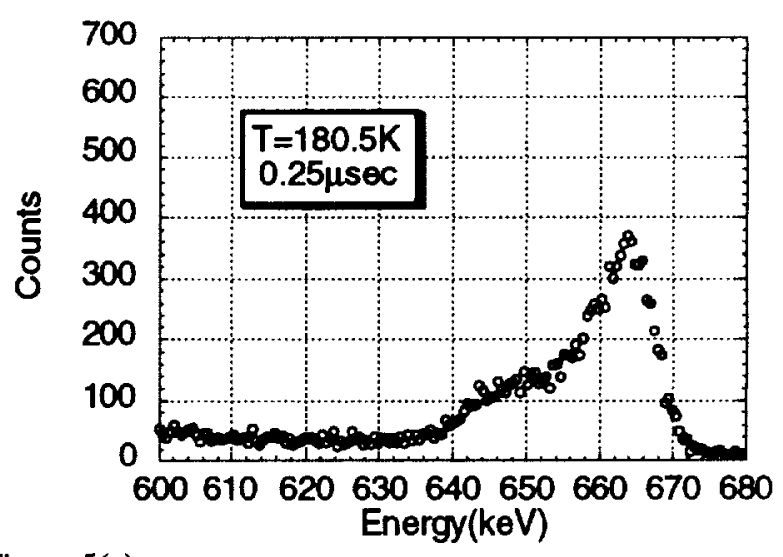

Figure 5(c)

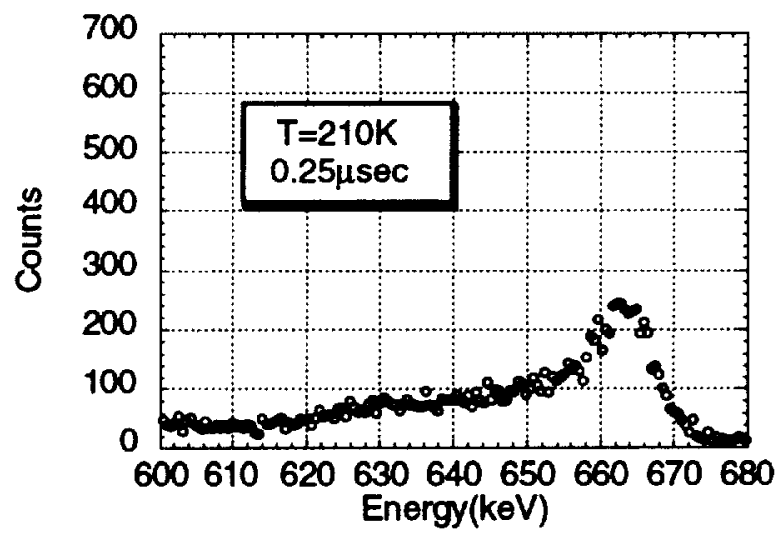

Figure 5(d)

Based on results to date, further improvements are possible in detector noise performance. Examples of these are refinements in preamplifier FET selection and input circuit. In addition, enhancements in the device charge collection may be realized though modifications to the detector processing. For example, fabrication and test of $\mathrm{Si}(\mathrm{Li})$ devices using a new phosphorus diffusion technique may remove charge trapping centers [3]. To fully understand the trapping which appears as a function of temperature we plan to examine the $\mathrm{Si}(\mathrm{Li})$ stacks with an analytical technique such as Deep Level Transient Spectroscopy.

In order to demonstrate spectrometer performance, we plan to readout individual device signals from the stack elements and apply previously developed detection algorithms to measurement of multi-line spectra [2]. 


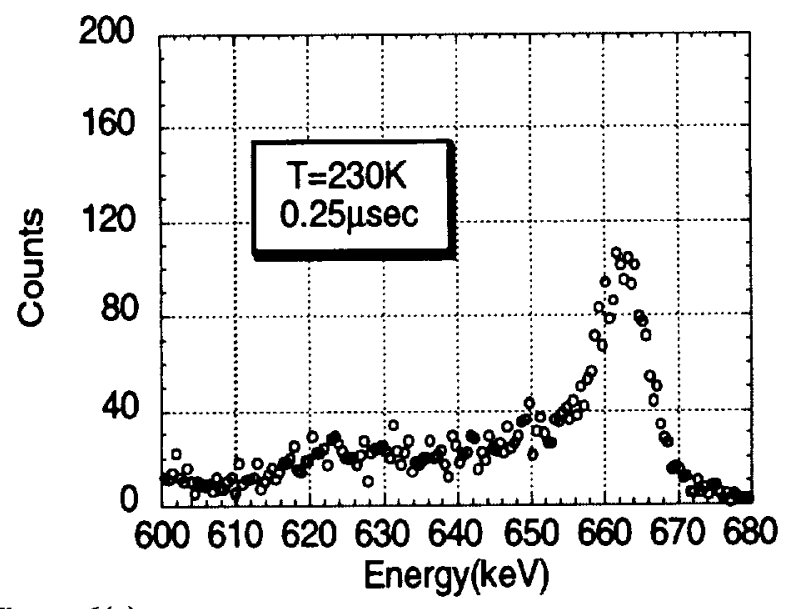

Figure 6(a)

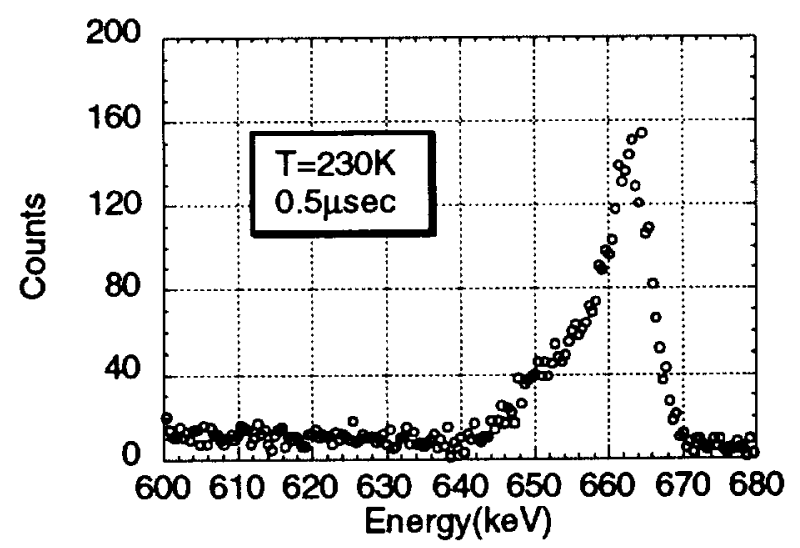

Figure 6(b)

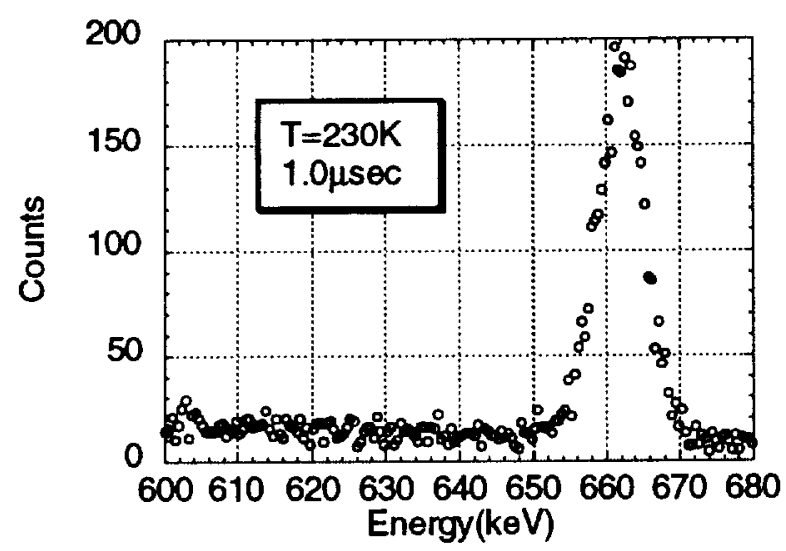

Figure 6(c)

Figure $6 .{ }^{137} \mathrm{Cs}$ photopeak shown for a single temperature,

\footnotetext{
as a function of $\tau_{\mathrm{s}}$. Single detector \#6454.
}

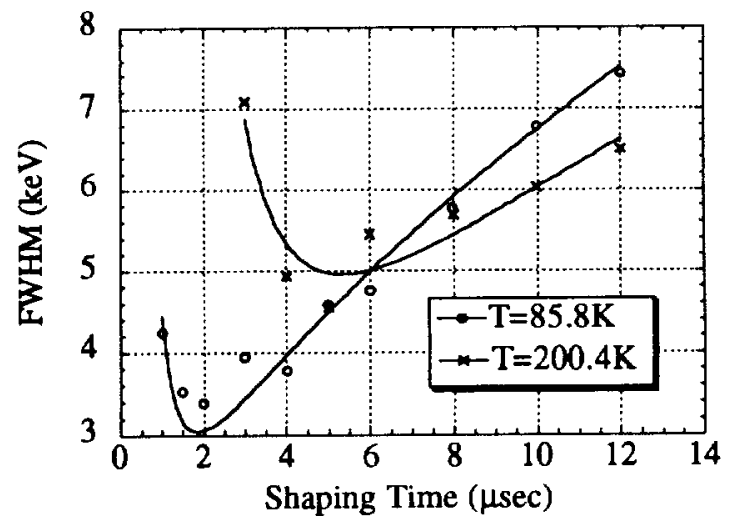

Figure 7. FWHM vs. amplifier shaping time for a $2 \mathrm{~cm}$ $\mathrm{Si}(\mathrm{Li})$ stack. Optimum $\tau_{\mathrm{s}}$ for minimum FWHM shifts by $>2$ times from $85 \mathrm{~K}$ to $200 \mathrm{~K}$. Curves are shown as an aid to the reader only.

\section{REFERENCES}

(1) G. Scott Hubbard, et al, IEEE Trans. Nuclear Science Vol. 39, Proceedings of IEEE 1991 NS Symposium, pp. 981-986 (1992).

(2) Robert E. McMurray, Jr., et al, IEEE Trans. Nuclear Science Vol. 40, Proceedings of IEEE 1992 NS Symposium, pp. 882889 (1993).

(3) J.T. Walton, N. Derhacobian, Y.K. Wong, and E.E. Haller, Applied Physics Letters, in press. 
